\title{
Formation of Mitochondrial Spheroid in Response to Oxidative Stress
}

\author{
Ming Li ${ }^{1,}$ Michael P. Goheen ${ }^{2}$ and Xiao-Ming Yin ${ }^{2},{ }^{3}$ \\ 1. Department of Pathology and Laboratory Medicine, Indiana University School of Medicine, \\ Indianapolis, IN, USA. Department of Pharmacology and Toxicology, School of Pharmaceutical \\ Sciences, Sun Yat-sen University, Guangzhou 510006, China \\ 2. Department of Pathology and Laboratory Medicine, and ${ }^{3}$ Department of Medicine, Indiana University \\ School of Medicine, Indianapolis, IN, USA
}

Mitochondrial are dynamic organelles with changing morphology consistent with their physiological and pathological conditions [1]. Mitochondria are double-membraned with cristae lying in the intermembrane space and the matrix enclosed within the inner membranes. Mitochondrial dynamics involve the change of membranes in these compartments and are controlled by specific molecular mechanisms. The molecular mechanisms for mitochondrial fission and fusion have been extensively studied, which are conserved from the yeast to the mammals [1]. However, much has yet to be learnt about the curvature and vesicular transformation about the mitochondria. This study investigated the dynamics of mitochondria under oxidative stress.

We have found that mitochondrial treated with carbonyl cyanide m-chlorophenylhydrazone (CCCP) underwent significant morphological changes, which could be defined by electron microscopy [2]. This change is characterized by the compression of one segment of the mitochondrial membranes, which may then allow the membrane to encircle the cytosol to form a phagosome-like structure, names as mitochondrial spheroid (Fig. 1). Notable, while the lumen contains various cytosolic materials, it is still open to the cytoplasm through a small orifice. The significance of this opening is not clear. The threedimensional structure of the mitochondrial spheroid has been confirmed by electron tomography [2]. Mitochondrial spheroids can localize with markers of the acidic compartment and can thus become acidified. Indeed, a limited proteolysis is detected, which mainly involves proteins located at the intermembrane space and inner membranes. It is possible that the outer membranes of the mitochondrial spheroids could fuse with the lysosome, which then delivers the hydrolases into the inter membrane space.

We have explored the molecular machinery involved in the formation of mitochondrial spheroids and find that mitofusins, Mfn1 and Mfn2, are required, whereas Parkin is inhibitory to this process [3]. This is mechanistically linked since Parkin can promote the degradation of the mitofusins following CCCP treatment. On the other hand, Parkin is required for CCCP-induced mitophagy [4]. Because of the dual effect of Parkin, it seems that cells expressing this molecule at a sufficient level will not generate mitochondrial spheroids, but will have mitophagy, following CCCP treatment. Thus a plausible explanation for the dual effect of Parkin in mitophagy and mitochondrial spheroid formation is that Parkin promotes mitofusin degradation and thus inhibits mitochondrial spheroid formation, which then allows the mitophagy to occur. It is thus possible that CCCP-induced mitochondrial spheroid may prevent mitochondria from mitophagy.

We have also defined another important regulator for mitochondrial spheroid formation, which is the reactive oxygen species (ROS) [3]. CCCP induces ROS, which promote mitochondrial fragmentation. It seems that mitochondrial fragmentation is a pre-requisite for mitochondrial spheroid formation since 
anti-oxidants could inhibit both phenomena. ROS are frequently generated following mitochondrial damage. Indeed, another mitochondrial toxin, sodium azide, can cause mitochondrial spheroid formation. Furthermore, acetaminophen overdose causes severe liver injury due to massive oxidative stress caused by mitochondrial damage. We have found that mitochondrial spheroids could be clearly induced under this condition, indicating that this phenomenon is potentially involved in the pathogenesis of oxidative liver injury caused by this drug.

In summary, the formation of mitochondrial spheroid reflects a novel mitochondrial dynamics that utilizes the molecular machinery involved in mitochondrial fusion and in mitophagy, suggesting that these events are closely coupled. The pathophysiological significance of this new dynamics has yet to be defined but it is possible that it is related to aging and oxidative mitochondrial damage.

\section{References:}

[1] B Westermann Nat. Rev. Mol. Cell Biol. 11 (2010) p.872

[2] W-X Ding et al, J. Bio. Chem. 287 (2012) p.42373

[3] W-X Ding et al, J. Bio. Chem. 287 (2012) p.42379

[4] R. J. Youle and D. P. Narendra, Nat Rev. Mol. Cell Biol. 12 (2011) p.9

[5] The authors acknowledge the funding from National Institute of Health (NIH R01CA83817 and R01 CA111456). Dr. Wen-Xing Ding (KUMC) is thanked for his contribution to this work.

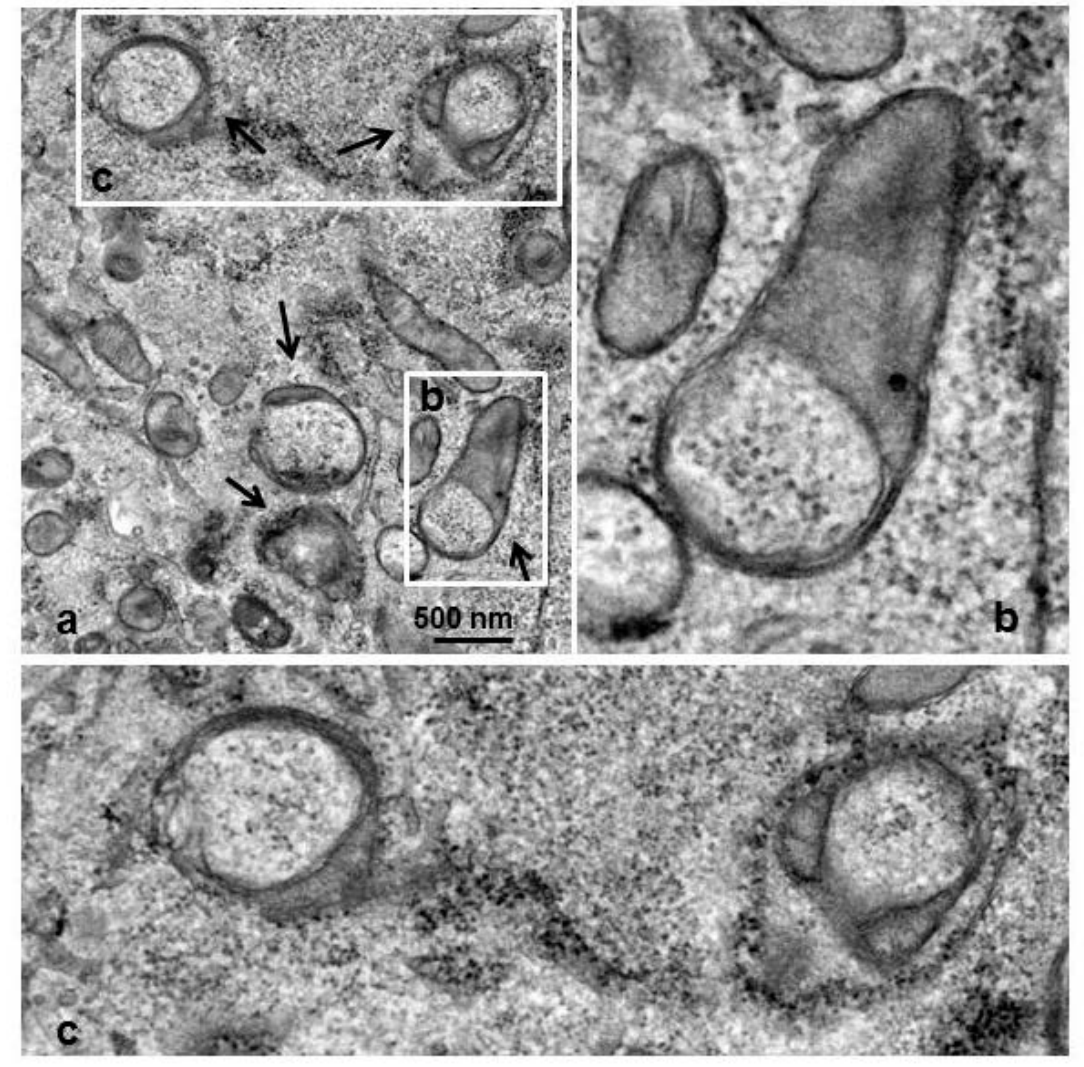

Figure 1. Murine embryonic fibroblasts were treated with CCCP $(30 \mu \mathrm{m})$ for 6 hours and then subjected to electron microscopic analysis. Arrows indicated mitochondrial spheroids. Examples in box $b$ and box $c$ are enlarged digitally in panel $\mathrm{b}$ and panel $\mathrm{c}$, respectively. Note that for each mitochondrial spheroid, a segment of the membranes is compressed and encircles the cytoplasm to form a phagosome-like structure with an internal lumen, which, however, still connects to the cytoplasm. 